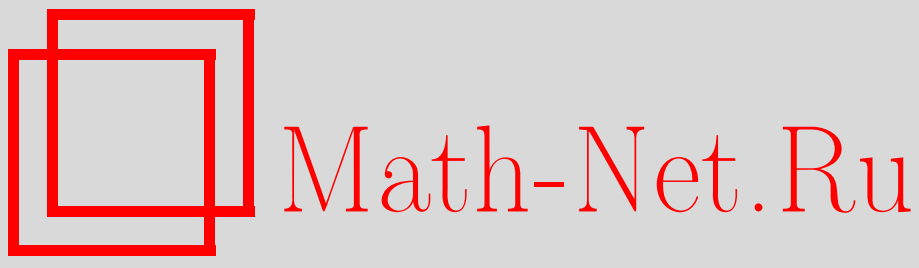

М. А. Штанько, Теорема А. А. Маркова и алгоритмически нераспознаваемые комбинаторные многообразия, Изв. РАН. Сер. матем., 2004, том 68, выпуск 1, 207-224

DOI: https://doi.org/10.4213/im471

Использование Общероссийского математического портала Math-Net.Ru подразумевает, что вы прочитали и согласны с пользовательским соглашением http://www . mathnet.ru/rus/agreement

Параметры загрузки:

IP: 3.85 .7 .115

26 апреля 2023 г., $13: 50: 28$ 


\title{
Теорема А. А. Маркова и алгоритмически нераспознаваемые комбинаторные многообразия
}

\begin{abstract}
Доказана теорема А. А. Маркова о существовании для любого $n \geqslant 4$ алгоритмически нераспознаваемого комбинаторного $n$-мерного многообразия. Указано конкретное многообразие, которое алгоритмически не распознается (ранее это не было сделано). Усиленная теорема Маркова доказана с помощью комбинаторных методов регулярных окрестностей и теории ручек. Доказательства одинаковы для любых $n \geqslant 4$. Используется группа Борисова [8] с неразрешимой проблемой тождества, имеющая две образующие и двенадцать соотношений. Именно применение группы Борисова явилось основой для доказательства усиленной теоремы Маркова (этим автор обязан С.И. Адяну).
\end{abstract}

Библиография: 26 наименований.

\section{Введение}

В 1958 г. А.А. Марков доказал алгоритмическую неразрешимость проблемы комбинаторного гомеоморфизма для комбинаторных многообразий размерности больше трех. Свои результаты А. А. Марков опубликовал в серии заметок [1]-[3], но в них доказательства были довольно краткими. Идеи Маркова реализовали В. В. Бун, В. Хакен и В. Поэнару, которые в 1968 г. доказали полную и даже несколько усиленную теорему Маркова [5]. Кроме того, они распространили теорему Маркова для гладких многообразий, указав, как с помошью локальных карт задавать гладкие многообразия эффективно. Однако работа [5] была сложной и перегруженной понятиями из логики и теории алгоритмов, тогда как Марков опирался на достаточно четкие геометрические рассуждения и даже предвосхитил некоторые конструкции, положившие начало новым понятиям теории перестроек на многообразиях (см. [6], [7] и др.).

Отметим, что ни в работах [1]-[3], ни в работе [5] не дан ответ на вопрос: какое же конкретное многообразие алгоритмически не распознается? (Указывалось только на то, что оно односвязное.)

В настоящей работе приведено доступное и полное доказательство теоремы Маркова. Мы будем рассматривать кусочно линейные многообразия и стремиться использовать как можно более простые геометрические и топологические конструктивные средства. Конкретную конструкцию алгоритмически нераспознаваемого многообразия построим, пользуясь простым примером группы с неразрешимой проблемой тождества, приведенным В.В. Борисовым в [8]. Этим многообразием является $16 \#\left(S^{2} \times S^{n-3}\right), \quad n \geqslant 5$; здесь символ \# обозначает

Работа выполнена при финансовой поддержке Российского фонда фундаментальных исследований (грант № 02-01-00803), Фонда поддержки научных школ (грант № НШ2185.2003.1), Программы ОМН РАН "Современные проблемы теоретической математики". 
операцию связной суммы многообразий одинаковой размерности: $M^{n} \# N^{n}$, а число 16 означает, что нужно взять 16 слагаемых.

В доказательстве основной теоремы настоящей работы (теоремы 3 ) использованы идеи и приемы, введенные самим А. А. Марковым. В частности, использован прием добавления к многообразию, построенному по данному групповому представлению $\mathrm{c} r$ образуюшими, $r$ тривиальных ручек индекса два.

В односвязном случае возникает стандартное многообразие размерности не менее $5 \quad\left(D^{n} \cup(m\right.$ ручек индекса два $\left.)\right)$, лежащее в $\mathbb{R}^{n}$. В рассматриваемом случае $m=16$. Граница этого многообразия является PL-многообразием $16 \#\left(S^{2} \times S^{n-3}\right), n \geqslant 5$. Это поясняет сформулированную в $\S 5$ теорему 3 .

В отличие от того, как строились многообразия самим Марковым, рассматриваемое многообразие строится как регулярная окрестность полиэдра группы, вложенного в евклидово пространство. Это позволяет не использовать теорему об односвязном $h$-кобордизме в кусочно линейной категории, которая, как хорошо известно, в этом случае неверна [13]-[15].

Представленное доказательство имеет комбинаторньй, а не топологический характер, и тем самым оно соответствует к алгоритмической постановке задачи.

Автор благодарен С.И. Адяну, А.В. Чернавскому и С. В. Матвееву за помощ и внимание к работе, а также А. Б. Скопенкову, П. М. Ахметьеву за ценные советы.

\section{§1. Предварительные сведения из теории групп и теории алгоритмов}

Через $\left\langle a_{1}, a_{2}, \ldots, a_{r} ; R_{1}, R_{2}, \ldots, R_{m}\right\rangle$ обозначим представление (задание) группы конечным числом образуюших $a_{1}, a_{2}, \ldots, a_{r}$ и конечным числом соотношений $R_{1}=1, R_{2}=1, \ldots, R_{m}=1$. Система образующих называется алфавитом, а выражения $R_{i}=1, i=1, \ldots, m,-$ определяющими соотношениями. Каждое соотношение записывается в виде слова в указанном алфавите. К буквам алфавита добавляются обратные буквы $a_{1}^{-1}, a_{2}^{-1}, \ldots, a_{r}^{-1}$. Слово, таким образом, представляется в виде конечной последовательности букв алфавита, каждая в степени 1 или -1 .

Будем обозначать через $\Pi(r, m)$ класс всех представлений, имеющих $r$ образующих и $m$ соотношений.

ТЕОРема АДяна-РАБИНА [9]-[12]. Если существует задание группы с неразрешимой проблемой тождества, то для некоторой пары чисел $(r, m)$, $r<m$, имеется класс представлений из $\Pi(r, m)$, для которого не существует алгоритма, распознающего представление тривиальной группь.

Рассмотрим группу Борисова [8] с неразрешимой проблемой тождества, которая имеет представление с двумя образуюшими и двенадшатью соотношениями:

$$
\left\langle a, b ; F_{i}=1, i=1,2, \ldots, 12\right\rangle .
$$

Заметим, что группа Борисова по построению имеет 5 образуюших и 12 соотношений, но согласно теореме HNN из [22] можно считать, что она имеет 2 образуюших и 12 соотношений. 
Введем две новые формальные образующие $t, s$, и пусть переменная $w$ принимает значения из некоторого рекурсивно-перечислимого набора слов группы Борисова. Тогда

$$
\left\{G_{w}\right\}=\left\langle a, b, t, s ; F_{i}=1, i=1,2, \ldots, 12, t w=w t^{2}, b t=t b^{2}, s w=w s^{2}, a s=s a^{2}\right\rangle
$$

- рекурсивно-перечислимый счетный набор групп представлений, каждое из которых имеет 4 образуюших и 16 соотношений.

Теорема Адяна-Рабина утверждает, что в классе представлений $\left\{G_{w}\right\} \subset$ $\Pi(4,16)$ алгоритмически неразрешима проблема тривиальности (см. [11]). Представления из класса $\left\{G_{w}\right\}$ будем записывать следуюшим образом:

$$
\left\langle t_{1}, t_{2}, t_{3}, t_{4} ; F_{i}=1, i=1,2, \ldots, 16\right\rangle \text {. }
$$

\section{§2. Краткие сведения из теории комбинаторных многообразий}

В этом параграфе напомним основные понятия и результаты из области кусочно линейных многообразий [16]. Используем сокращение PL для слов "кусочно линейньй”. Евклидово пространство размерности $n$ с естественной линейной, $\mathrm{a}$ также с естественной PL-структурой обозначим $\mathbb{R}^{n}$, его начало - $O$. Стандартный куб обозначим $\mathbb{B}^{n}$, его гранищу, являющуюся сферой размерности $n-1$ со стандартной PL-структурой, обозначим $\mathbb{S}^{n-1}$. Изоморфный PL-образ $\mathbb{B}^{n}$ будем обозначать через $D^{n}$ или $Q^{n}$, а PL-образ сферы $\mathbb{S}^{n-1}-$ через $S^{n-1}$ с различными индексами. Через $K$ обозначим конечный симплициальный комплекс и этой же буквой обозначим полиэдр, являющийся телом этого комплекса.

Определим операцию симплициального стягивания в симплициальном комплексе $K$. Пусть в комплексе $K$ существует такая пара открытых симплексов $A^{k}$ и $A^{k-1}$, что $A^{k-1}$ является гранью $A^{k}$ и только $A^{k}$. Тогда в комплексе $K$ определена операция

$$
K \searrow\left(K \backslash\left(\operatorname{Int} A^{k} \cup \operatorname{Int} A^{k-1}\right)\right)=K_{1},
$$

называемая симплиииальным стязиванием комплекса $K$ в $K_{1}$. Это означает, что мы удаляем из комплекса $K$ открытый симплекс $A^{k}$ и его открытую грань $A^{k-1}$. Комплекс $K$ комбинаторно стязивается на свой подкомплекс $L$, если $L$ получается в результате конечного числа симплищиальных стягиваний. В этом случае условимся писать $K \searrow L$.

Кусочно линейным многообразием $M^{n}$ (PL-многообразием) размерности $n$ называется такой полиэдр, что звезды вершин любой его триангулящии PL-эквивалентны симплексу размерности $n$. Если точка переходит внутрь симплекса при некотором таком PL-изоморфизме, то она называется внутренней точкой многообразия, в противном случае - точкой края. Это определение, очевидно, инвариантно.

Пусть $P$ - подкомплекс в PL-многообразии $M^{n}$; тогда абсолютной регулярной окрестностью полиэдра $P$ в многообразии $M$ назьвается компактное PL-многообразие $N^{n}$ с краем $\partial N^{n}$ такое, что Int $M^{n} \supset N^{n} \supset \operatorname{Int} N^{n} \supset P$ и $N^{n} \searrow P$.

Еще в 1939 г. теорию регулярных окрестностей изучал Дж. Г. К. Уайтхед [20]. Он доказал, помимо многих других важных теорем, следуюшие теоремы.

Tеорема W1 (существование). Пусть $N$ - PL-комплекс, покрывающий PL-многообразие $M$, и $K$ - его подкомплекс. Тогда звезда комплекса $K$ во втором барицентрическом подразделении комплекса $N$ является (абсолютной) регулярной окрестностью: $\operatorname{St}\left(K, N^{\prime \prime}\right) \searrow K$. 
ТЕОРЕма W2 (единственность). Для любых двух регулярных окрестностей подкомплекса $K$ в PL-многообразии $M$ существует PL-гомеоморфизм многообразия $N$, тождественный на $K$ и переводящий одну из них на другую.

ТЕОРема W3. Если PL-многообразие $M^{n}$ с границей Ә ${ }^{n}$ комбинаторно стягивается в точку, то $M^{n}$ - кусочно линейная клетка размерности $n$.

Используя комбинаторную технику Зимана, будем рассматривать полиэдр $P$, разбитый на клетки, т. е. некоторую конечную совокупность выпуклых многогранников, при этом:

а) все грани любого вьпуклого многогранника из этой совокупности являются клетками;

б) пересечение выпуклых многогранников - тоже клетка, входящая в эту совокупность клеток.

Полиэдры $P$ с такой структурой разложения на выпуклые многогранники будем называть клеточными полиэдрами (в отличие от симплициальных полиэдров). Тогда элементарные операции удаления и добавления клеток рассматриваются точно так же, как и в симплициальной категории (см. [16]). Все результаты о регулярных окрестностях в этом новом смысле рассматриваются и верны точно так же, как и в симплищиальной категории.

Операция удаления клетки $A^{k}$ (комбинаторная стягиваемость) производится так же, как и в симплициальном случае, при этом удаляемая клетка должна иметь на своей поверхности свободную клетку $A^{k-1}$, т. е. инцидентную в полиэдре $P$ только той клетке размерности $k$, на поверхности которой она лежит. Тогда операция удаления двух открытых клеток

$$
P \searrow\left(P \backslash\left(\operatorname{Int} A^{k} \cup \operatorname{Int} A^{k-1}\right)\right)=P_{1}
$$

называется комбинаторным стягиванием полиэдра $P$ в $P_{1}$ и обозначается $P \searrow P_{1}$.

ЛЕМма 1. 1) Пусть многообразие $W$ является комбинаторным конусом симплиииального отображения $\Phi: \partial W \rightarrow V$, где $V$ - букет из грании четырех треугольников $(4 \bigvee \partial \Delta)$. $B$ таком случае многообразие $W$ получает естественную клеточную структуру: она состоит из симплексов, лехащих в $\partial W$, симплексов в $V$ (это точки и отрезки) иклеток $B_{\sigma}$, әде $\sigma$ - симплекс в $\partial W$. При әтом, как легко видеть, $B_{\sigma}$ является конусом симплициального отображсения $k$-симплекса $\sigma$ в отрезок или вершину.

Тогда $B_{\sigma}$ комбинаторно гомеоморфна клетке размерности $k+1$. Ясно, что если $\sigma_{1}$ - грань $\sigma$, то $B_{\sigma_{1}}$ лежит на граниче $B_{\sigma}$, и граница $\partial B_{\sigma}$ состоит из $\sigma, \Phi(\sigma)$ иклеток $B_{\gamma}$, где $\gamma \subset \partial \sigma$.

2) Если $K$ - подкомплекс триангулячии дW, то существует клеточное комбинаторное стягивание $W \searrow(V \cup C)$, где $C$ - конус отображения $\left.\Phi\right|_{K}$. В самом деле, такое стягивание есть последовательность удалений пар $\left(\sigma, B_{\sigma}\right)$, где $\sigma$ - один из симплексов в $\partial W \backslash K$, имеющих на данном шаге максимальную размерность.

Доказательство леммы очевидно (см. [16]). 


\section{§3. Многообразия Маркова и ручки индекса два}

Все конструкции в настояшей статье будут рассматриваться в евклидовом пространстве $\mathbb{R}^{n}, n \geqslant 5$. Пусть $M^{n}$ - замкнутые связные многообразия в $\mathbb{R}^{n}, \partial M^{n}$ их границы. В соответствии с этим будем называть многообразия $\partial M^{n}$ в $\mathbb{R}^{n}$ незаполненными многообразиями и $M^{n}$ в $\mathbb{R}^{n}$ - заполненными.

Нам понадобится класс П $(4,16)$ представлений груп, задаваемых $r=4$ образуюшими и $m=16$ соотношениями.

Как обычно, полиэдр, отвечающий представлению класса $\Pi(r, m)$, образуется из букета $r$ ориентированных окружностей "наворачиванием" на них (согласно $m$ данным соотношениям) гранищ двумерных клеток. Для этого граница диска делится на отрезки по числу вхождений в соотношение букв $a_{i}^{ \pm 1}$ и каждой букве отвечает монотонное отображение соответствующего отрезка на соответствующую окружность в направлении, отвечающем знаку показателя, равного показателю буквы $a$. После этого диск приклеивается к букету по получившемуся отображению его края.

Построенный по представлению $\pi \in \Pi(r, m)$ двумерный полиэдр $P^{2}(\pi)$ мы затем вкладываем в $\mathbb{R}^{n}, n \geqslant 5$, и рассматриваем его регулярную окрестность. Полученное заполненное многообразие обозначим $N^{n}\left(P^{2}(\pi)\right)=N^{n}(\pi)$. Хорошо известно, что при $n \geqslant 5$ фундаментальная группа двумерного комплекса изоморфна фундаментальной группе гранищы его регулярной окрестности.

Поскольку в настоящей работе полиэдр $P^{2}(\pi)$ играет важную роль, мы отметим некоторые свойства этого полиэдра.

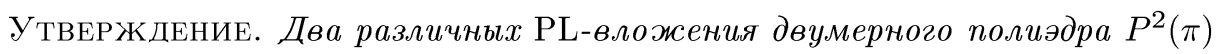
в $\mathbb{R}^{n}, n \geqslant 5$, имеют $\mathrm{PL}$-изоморфные регулярные окрестности.

Доказательство см. в [21].

Кусочно линейной ручкой индекса $k$ назьвается PL-произведение двух дисков $D^{k} \times_{\mathrm{PL}} D^{n-k}$ со следуюшей структурой. Центр диска $D^{n-k}$ обозначим $O$. Диск $D^{k} \times O$ называется остовным диском ручки, его край $\partial D^{k}-$ подошвенной сферой ручки. Произведение $\partial D^{k} \times_{\mathrm{PL}} D^{n-k}$ образует подошвенное многообразие ручки $D^{k} \times{ }_{\mathrm{PL}} D^{n-k}$, по которому она приклеивается к многообразию $M^{n}$. В дальнейшем нам понадобятся только ручки индекса два $D^{2} \times_{\mathrm{PL}} D^{n-2}$. В этом случае подошвенным многообразием этой ручки является PL-произведение $S^{1} \times_{\mathrm{PL}} D^{n-2}$, где $S^{1}=\partial D^{2}$ принадлежит краю $\partial M^{n}$ многообразия $M^{n}$.

В данной статье будем использовать специальное приклеивание ручки индекса два к многообразию $M^{n}$, которое назовем стандартным приклеиванием. Определим стандартное приклеивание геометрически. Рассмотрим поверхность трехмерного куба без одной двумерной грани и обозначим этот диск $\Pi^{2}$. Естественное $n$-мерное утолщение этого диска называется стандартной ручкой индекса два.

Построим это утолщение. Рассмотрим в $\mathbb{R}^{3}$ гиперплоскость $\mathbb{P}^{2}$ и три трехмерных куба $I_{1}^{3} \subset I_{2}^{3} \subset I_{3}^{3}$ таких, что одна грань $I_{i}^{2}$ куба $I_{i}^{3}$ лежит на $\mathbb{P}^{2}$, причем грани $I_{i}^{2}$ гомотетичны и $I_{1}^{2} \subset \operatorname{Int} I_{2}^{2} \subset I_{2}^{2} \subset \operatorname{Int} I_{3}^{2}$. Тогда $I_{3}^{3} \backslash \operatorname{Int} I_{1}^{3} \backslash \operatorname{Int} I_{1}^{2}$ есть трехмерная ручка $H^{3}$ индекса два с клеткой $\partial I_{2}^{3} \backslash\left(\operatorname{Int} I_{2}^{2}\right)=\Pi^{2}$ в качестве остовного диска, PL-изоморфная $\Pi^{2} \times_{\mathrm{PL}}[-1,+1]$. Умножив $H^{3}$ на $(n-3)$-мерньй куб $Q^{n-3}$ в ортогональной к $\mathbb{R}^{3}$ плоскости в $\mathbb{R}^{n}=\mathbb{R}^{3} \times \mathbb{R}^{n-3}$, получим стандартную ручку $\Pi^{2} \times{ }_{\mathrm{PL}} Q^{n-2}=H^{n}$ индекса два размерности $n$.

Пусть $B^{n-1}$ - гиперплоский диск размерности $n-1$, выбранный где-либо на гранище многообразия $N^{n}(\pi) \subset \mathbb{R}^{n}$ (регулярной окрестности полиэдра $P(\pi)$ ), по- 


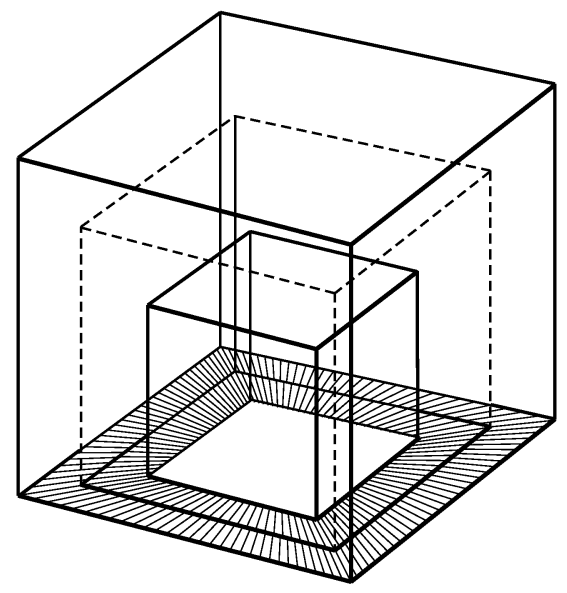

Рис. 1. Стандартная ручка $H^{n}=\Pi^{2} \times_{\mathrm{PL}} Q^{n-2}$

строенного, как и выше, по заданному представлению $\pi \in \Pi(4,16)$ и вложенного в $\mathbb{R}^{n}, n \geqslant 5$. Приклеим к диску $B^{n-1}$ указанным на рис. 1 образом с внешней стороны от $N^{n}(\pi)$ ручки $\Pi_{j}^{2} \times_{\mathrm{PL}} Q^{n-2}$ в количестве, равном $j=4$, которые попарно не пересекаются. Эти приклеивания назовем стандартными, полученное многообразие-заполненным многообразием Маркова:

$$
M^{n}(\pi)=N^{n}(\pi) \cup\left(\bigcup_{j=1}^{4}\left(\Pi_{j}^{2} \times_{\mathrm{PL}} Q^{n-2}\right)\right) .
$$

А. А. Марков определил в [3] многообразие, отвечающее данной $\Pi(r, m)$-группе, по-своему, используя некоторую конструкцию из книги [4]. Введенное определение многообразия Маркова достаточно для доказательства основной теоремы, и доказательство совпадения этих многообразий нам не понадобится.

Далее приведем обшее определение приклеивания ручки индекса два к подошвенной торической окрестности замкнутой ломаной $\Sigma^{1}$, лежащей на границе многообразия $M^{n} \subset \mathbb{R}^{n}$. Торическая окрестность ломаной $\Sigma^{1}$ представляет собой нормальную трубчатую PL-окрестность $\nu_{\mathrm{PL}}\left(\Sigma^{1} \times_{\mathrm{PL}} Q^{n-2}\right) \subset M^{n-1}$ над базойломаной окружностью $\Sigma^{1}$ - и со слоем $D^{n-2}$ в многообразии $M^{n-1}=\partial M^{n}$. Для приклеивания ручки необходимо, чтобы эта окрестность имела структуру прямого произведения и при этом было выбрано конкретное представление окрестности в виде прямого произведения $\Sigma^{1} \times_{\mathrm{PL}} D^{n-2}$, где $\Sigma^{1}=\Sigma^{1} \times O, O$ - центр диска $D^{n-2}$.

В рассматриваемых случаях такая структура будет возникать естественным образом, причем приклеиваемые ручки будут лежать в том же пространстве $\mathbb{R}^{n}$, что и многообразие $M^{n}$.

\section{§4. Окружности в односвязных многообразиях}

ЛЕмма 2. В односвязном $n$-мерном комбинаторном многообразии, $n \geqslant 4$, любие две ломаные окружности PL-изотопны.

ДокАЗАТЕЛЬСтво. Не нарушая общности, можно считать, что $n=4$. Пусть $M^{4}$ - связное, односвязное, замкнутое комбинаторное многообразие, и пусть $S_{1}^{1}$ 
и $S_{2}^{1}$ - две комбинаторные окружности в $M^{4}$, которые гомотопны в силу односвязности многообразия $M^{4}$. Достаточно показать, что если $S_{1}^{1}$ и $S_{2}^{1}$ не пересекаются, то сушествует PL-вложение $f$ цилиндра $\mathbb{S}^{1} \times[1,2]$ в $M^{4}$, при котором $\mathbb{S}^{1} \times i$ переходит в $S_{i}^{1}, i=1,2$, и которое не имеет особых точек (см. ниже).

Натягивая пленку гомотопии между окружностями $S_{1}^{1}$ и $S_{2}^{1}$ (т. е. рассматривая отображение $f\left(\mathbb{S}^{1} \times[1,2]\right) \rightarrow \operatorname{Int} M^{4}$, где $f: \mathbb{S}^{1} \times[1,2] \rightarrow M^{4}$ и $f\left(\mathbb{S}^{1} \times 1\right)=S_{1}^{1}$, $\left.f\left(\mathbb{S}^{1} \times 2\right)=S_{2}^{1}\right)$ и приводя ее в общее положение, получим, что пленка гомотопии превращается в симплициальную пленку с конечным числом ее (двойных) трансверсальных нульмерных самопересечений в $M^{4}$. Окрестность двойной точки самопересечения PL-эквивалентна окрестности точки пересечения двух ортогональных дисков. Используя "ріре"-лемму Зимана, можно устранить эти нульмерные сингулярности (их конечное число) следуюшим образом: точка пересечения двух дисков соединяется ломаной дугой с краем пленки так, что дуга не содержит остальные точки самопересечения. Затем один из дисков заменяется двумерной трубкой, которая образована следом края этого диска и самим диском в конечный момент параллельного переноса вдоль указанной дуги.

Аналогичные операции проводятся для всех двойных трансверсальных точек самопересечения независимо, и, таким образом, пленка превращается в кусочно линейный двумерный цилиндр, натянутый без самопересечений между окружностями $S_{1}^{1}$ и $S_{2}^{1}$ и лежащий в $\operatorname{Int} M^{4}$. Обозначим построенньй цилиндр с краями $S_{1}^{1}$ и $S_{2}^{1}$ через $Z$.

Далее мы должны устранить точки построенного цилиндра $Z$, не являющиеся локально плоскими. Нетрудно видеть, что не локально плоские точки могут быть только вершинами триангуляции построенного цилиндра $Z$. Пусть точка $p$ является не локально плоской вершиной. Это означает, что если мы построим малую трехмерную сферу $\mathbb{S}^{3}$ с центром в точке $p$, то пересечение $\operatorname{lk}(p, Z)$ трехмерной сферы с цилиндром будет заузленной в ней окружностью $C^{1}$.

Таким образом, мы имеем трехмерную сферу $\mathbb{S}^{3} \subset M^{4}$ и в этой трехмерной сфере заузленную окружность $C^{1}$. Часть цилиндра $Z$, ограниченную окружностью $C^{1}$, мы удаляем. Вместо этой части к кусочно линейной окружности $C^{1}$ приклеиваем погруженную локально плоскую клетку $D^{2}$. Обозначим через $D^{4}$ клетку в $M^{4}$, ограниченную сферой $\mathbb{S}^{3}, \partial D^{4}=\mathbb{S}^{3}$.

Кусочно линейную клетку $D^{2}$ можно предполагать локально плоской и без самопересечений, так как ломаная окружность $C^{1}$ не заузливает в $\mathbb{R}^{4}$. Теперь приводим в общее положение уменьшенный цилиндр $Z \backslash \operatorname{Int} D^{4}$ и приклеенный к нему по окружности $C^{1}=Z \cap D^{4}$ кусочно линейньй диск $D^{2}$.

Можно считать, что в точках склейки на $C^{1}$ объединение $\left(Z \backslash \operatorname{Int} D^{4}\right) \cup D^{2}$ является локально плоским и что объединение $Z \cup D^{2}$ состоит из конечного числа двойных трансверсальных точек, которые мы удаляем, как указано выше, используя "ріре"-лемму Зимана.

Получившийся в итоге цилиндр $Z$ с краями $S_{1}^{1}, S_{2}^{1}$ является поверхностью с краем в $M^{4}$ без локальных узловых особенностей. Следовательно, PL-изотопию окружности $S_{1}^{1}$ в $S_{2}^{1}$, проводимую по цилиндру $Z$, можно продолжить до PL-изотопии во всем многообразии $M^{4}$. Лемма доказана. 


\section{§5. Основные результаты}

В этом параграфе каждому представлению $\pi$ из класса $\left\{G_{w}\right\}$ сопоставим заполненное многообразие Маркова $M^{n}(\pi)$. Докажем, что фундаментальная группа многообразия $M^{n}(\pi)$ тривиальна тогда и только тогда, когда граница этого многообразия PL-гомеоморфна многообразию $16 \#\left(\mathbb{S}^{2} \times \mathbb{S}^{n-3}\right), n \geqslant 5$. Отсюда с помощью теоремы Адяна-Рабина выводится, что многообразие $16 \#\left(\mathbb{S}^{2} \times \mathbb{S}^{n-3}\right), n \geqslant 5$, алгоритмически не распознается.

ТЕОРема 1. Предположим, что $\pi \in\left\{G_{w}\right\}-$ представление, которое задает тривиальную группу. Тогда соответствующее многообразие Маркова $M^{n}(\pi)$ имеет край, $\mathrm{PL-гомеоморфный} \mathrm{многообразию} 16 \#\left(\mathbb{S}^{2} \times \mathbb{S}^{n-3}\right)$, $n \geqslant 5$.

ДокАЗАТЕЛЬСтво. 1) Построим многообразие $\bar{W}^{n}(\pi)$ и докажем, что оно PL-изоморфно $N^{n}(\pi)$.

а) Построим крендель $W^{n}(V)$.

Сначала выберем в пространстве $\mathbb{R}^{n}$ плоскость $\mathbb{R}^{2}$. Рассмотрим вложение букета $V$ из 4 -х окружностей в плоскости $\mathbb{R}^{2}$ в виде правильного букета из гранищ $\Sigma_{j}$ равных равносторонних треугольников $\Delta_{j}, j=1, \ldots, 4$, с обшей вершиной $O$ и равными углами между ними.

Построим (см. рис. 2) регулярную окрестность $N^{2}(V)$, где $V=\bigcup_{j=1}^{4} \Sigma_{j}$ (далее индекс $j$ циклически меняется от 1 до 4).

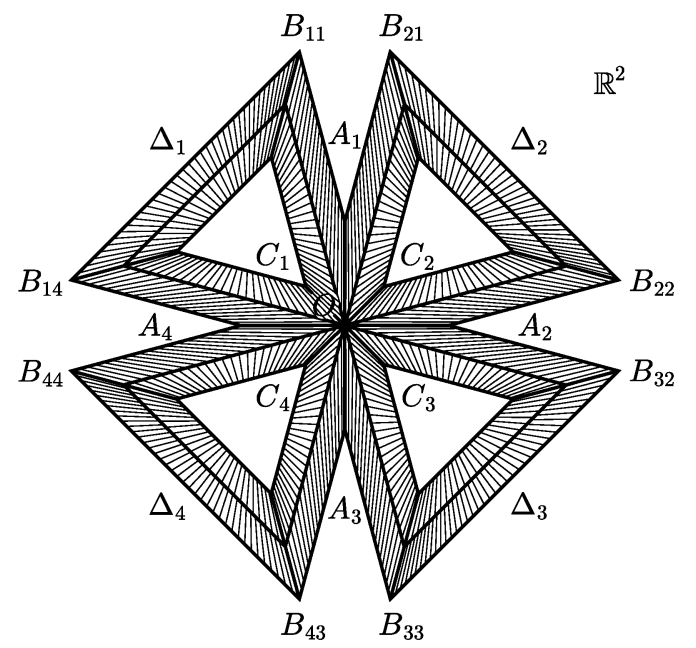

Рис. 2. Регулярная окрестность $N^{2}(V)$ букета $V$

Построим отрезок $O A_{1}$ биссектрисы угла между $\Delta_{1}$ и $\Delta_{2}$, длина которого равна половине стороны треугольников. Далее так же строим отрезки $O A_{j}$ биссектрис между треугольниками $\Delta_{j}$ и $\Delta_{j+1}$.

Для вершин треугольника $\Delta_{1}$, отличных от $O$, возьмем на продолжениях биссектрис его углов точки $B_{14}$ и $B_{11}$ и аналогично для треугольников $\Delta_{j}$ - точки $B_{j, j-1}$ и $B_{j j}$ так, что отрезки $A_{j-1} B_{j, j-1}, B_{j, j-1} B_{j j}, B_{j j} A_{j}$ параллельны соответствуюшим сторонам треугольника $\Delta_{j}$ (индекс $j$ берется по модулю 4 ). Кроме 
того, внутри каждого треугольника $\Delta_{j}$ рассмотрим гомотетичный ему треугольник $\widetilde{\Delta}_{j}$ со стороной, равной $1 / 3$ стороны внешнего треугольника $\Delta_{j}$.

Обозначим через $L$ область, ограниченную ломаной

$$
A_{1} B_{21} B_{22} A_{2} B_{32} B_{33} A_{3} B_{43} B_{44} A_{4} B_{14} B_{11} A_{1} \text {. }
$$

Тогда $L \backslash\left(\bigcup_{j=1}^{4} \operatorname{Int} \widetilde{\Delta}_{j}\right)$ представляет собой регулярную окрестность букета $V$, которую мы обозначили $N^{2}(V)$. Четыре отрезка, соединяюшие $O$ с ближайшими вершинами $C_{j}$ треугольников $\widetilde{\Delta}_{j}$, четыре отрезка $O A_{j}$ и отрезки, проведенные через вершины $B_{k l}, 1 \leqslant k \leqslant 4,1 \leqslant l \leqslant 4$, к центрам треугольников, вместе с букетом $V$ разбивают $N^{2}(V)$ на $4 \times 6$ четырехугольника, каждый из которых можно представить в виде прямого произведения одной из сторон $\Delta_{j}$ на отрезок. В результате можно представить $N^{2}(V)$ в виде объединения $(V \backslash O) \times[-1,1]$ с восемью отрезками, имеющими общий конец $O$. Здесь 1 отвечает точкам на $\partial L$, $O$ - точкам на $V$ и -1 - точкам на $\bigcup\left(\partial \widetilde{\Delta}_{j} \backslash C_{j}\right)$.

Букет $V$ разбивает $N^{2}(V)$ на две части. Внешняя часть PL-гомеоморфна произведению $\partial L \times[0,1]$ со склеенными четырьмя концами четырех отрезков $\left[A_{j}, O\right]$, $j=1, \ldots, 4$. Внутренняя часть PL-гомеоморфна $\left(\bigcup_{j=1}^{4} \partial \widetilde{\Delta}_{j}\right) \times[-1,0]$ также со склеенными четырьмя точками, лежашими на концах четырех отрезков $\left[C_{j}, O\right]$, $j=1, \ldots, 4$.

Итак, мы построили регулярную окрестность $N^{2}(V)$ в плоскости $\mathbb{R}^{2}$. Рассмотрим теперь в пространстве $\mathbb{R}^{n}, n \geqslant 5$, плоскость $\mathbb{R}^{n-2}$, ортогональную плоскости $\mathbb{R}^{2}$ в точке $O$. Пусть $Q^{n-2}-$ куб с центром в $O$, находящийся в плоскости $\mathbb{R}^{n-2}$. Обозначим через $W^{n}(V)$ прямое произведение $N^{2}(V) \times Q^{n-2}$, которое является регулярной окрестностью букета $V$ в пространстве $\mathbb{R}^{n}$. Нетрудно доказать, что $N^{2}(V) \times Q^{n-2}-\mathrm{PL}$-многообразие с краем и $V$ лежит внутри этого многообразия. Нетрудно также, используя комбинаторную стягиваемость $Q$ в точку, построить комбинаторную стягиваемость $N^{2}(V) \times Q^{n-2} \searrow V$.

Будем называть $W^{n}(V)$ крендельным многообразием над букетом из четырех окружностей $V=\bigcup_{j=1}^{4} \Sigma_{j}$, или просто кренделем.

б) Построим полоски, отвечающие соотношениям $F_{i}$ представления $\pi$. Обозначим через $E$ объединение восьми отрезков $\left[O, A_{j}\right]$ и $\left[O, C_{j}\right], j=1, \ldots, 4$. Тогда

$$
W^{n}(V)=\left((V \backslash O) \times Q^{n-1}\right) \cup\left(E \times Q^{n-2}\right), \quad Q^{n-1}=Q^{n-2} \times[-1,1] .
$$

Гранища $\partial W^{n}(V)$ представляется в виде объединения

$$
\left((V \backslash O) \times \partial Q^{n-1}\right) \cup \partial\left(E \times Q^{n-2}\right),
$$

где $\partial\left(E \times Q^{n-2}\right)=\left(E \times \partial Q^{n-2}\right) \cup\left(\partial E \times Q^{n-2}\right)$. Здесь $\partial E$ есть восемь точек $\bigcup_{j=1}^{4}\left(A_{j} \cup C_{j}\right)$.

Заметим, что для каждой точки $x$ на $\partial W^{n}(V)$ однозначно определен отрезок $[x, y]$, где $y \in V$. Если $x \in \partial\left(E \times Q^{n-2}\right)$, то $y=O$ и отрезок $[x, y]$ однозначно определен. Если же $x \in \partial\left((V \backslash O) \times Q^{n-1}\right)$, то $y$-центр PL-диска $y \times Q^{n-1}$, границе которого принадлежит точка $x$, и $[x, y]$ - радиус этого диска. Обозначим полученное отображение через $\Phi: x \mapsto y$. Отрезки $[x, y]$ образуют, очевидно, непрерывное семейство, покрывающее крендельное многообразие $W^{n}(V)$. Это семейство устроено так, что один конец каждого отрезка $[x, y]$ - точка $x$ - принимает значения из многообразия $\partial W^{n}(V)$, а другой конец - точка $y$ - из $V$, причем эти отрезки $[x, y]$ могут пересекаться только в своих концах $\{y\}$. В силу приведенного свойства се- 
мейства $[x, y]$ определена ретракция $\Phi:[x, y] \mapsto y$ всего кренделя $W^{n}(V)$ на $V$, совпадающая с $\Phi$ на $\partial W^{n}, \Phi: W^{n}(V) \rightarrow V$.

Таким образом, если $K$ - любой полиэдр на $\partial W^{n}(V)$, то ограничение отображения $\Phi$ на $K$ есть PL-отображение и объединение отрезков $[x, y]$, отвечающих точкам на $K$, является конусом этого отображения $\left.\Phi\right|_{K}$.

Пусть теперь $\pi \in \Pi(4,16)$ - одно из представлений множества $\left\{G_{w}\right\}$ с образующими $t_{j}, 1 \leqslant j \leqslant 4$, и соотношениями $F_{i}, 1 \leqslant i \leqslant 16$. Пусть $F_{i}=t_{i 1}^{\varepsilon_{1}^{i 1}} \cdots t_{i s}^{\varepsilon^{i s}}$, $\varepsilon^{i p}= \pm 1,-$ одно из соотношений. Существует каноническое отображение $\varphi_{i}$ окружности $\mathbb{S}_{i}^{1}$ в букет $V, \varphi_{i}: \mathbb{S}_{i}^{1} \rightarrow V, 1 \leqslant i \leqslant 16$, отвечающее этому соотношению. Разобьем $\mathbb{S}_{i}^{1}$ на $s$ равных частей (дуг) $l_{i p}$ и каждую часть - еше на три равные дуги. Отобразим $l_{i p}$ на край $\Sigma_{i}$ треугольника $\Delta_{i}$, последовательно переводя каждую из трех дуг в $l_{i p}$ на соответствующую сторону треугольника и выбирая направление в зависимости от знака $\varepsilon^{i p}$.

Построим отображение $\widetilde{\varphi}_{i}:\left(\mathbb{S}_{i}^{1} \times[0,1]\right) \rightarrow W^{n}(V)$, продолжающее отображение $\varphi_{i}: \mathbb{S}_{i}^{1} \rightarrow V$ так, что $\widetilde{\varphi}_{i}\left(\mathbb{S}_{i}^{1} \times 1\right) \subset \partial W^{n}(V), \widetilde{\varphi}_{i}\left(\mathbb{S}_{i}^{1} \times O\right)=\varphi_{i}: \mathbb{S}_{i}^{1} \rightarrow V$. При этом потребуем, чтобы каждый отрезок $t \times[0,1], t \in \mathbb{S}_{i}^{1}$, линейно отображался на отрезок $[x, y]$, где $y=\varphi_{i}(t), x=\widetilde{\varphi}_{i}(t \times 1), y=\Phi(x)$.

Рассмотрим один из треугольников $\Delta_{j}, 1 \leqslant j \leqslant 4$. Прообраз $\Phi^{-1}\left(\Sigma_{j} \backslash O\right)$ есть прямое произведение $\left(\Sigma_{j} \backslash O\right) \times \mathbb{S}^{n-2} \subset \partial W^{n}(V), \mathbb{S}^{n-2}=\partial Q^{n-1}$.

Пусть $\varphi_{i}$ отображает дугу $l_{i p}$ на $\Sigma_{j} \backslash O$. Требуемое отображение на внутренние точки дуги $l_{i p}$ однозначно определяется отображением $l_{i p} \rightarrow \mathbb{S}^{n-2}, n \geqslant 5$. Можно потребовать, чтобы это отображение продолжалось на конщы $l_{i p}$ и продолжение отображало их в точки на $O \times \mathbb{S}^{n-3}, n \geqslant 5$.

Покажем, как для одной дуги $l_{i p}$ построить вложение Int $l_{i p} \rightarrow\left(\Sigma_{j} \backslash O\right) \times \mathbb{S}^{n-2}$ с требуемыми свойствами.

Пусть $P:\left(\Sigma_{j} \backslash O\right) \times \mathbb{S}^{n-2} \rightarrow \Sigma_{j} \backslash O$ - проекция прямого произведения. Напомним, что $\varphi_{i}$ отображает дугу $l_{i p}$ на $\Sigma_{j} \backslash O$. Если $q-$ произвольное отображение дуги Int $l_{i p}$ в $\mathbb{S}^{n-2}$, то отображение $\bar{q}: l_{i p} \rightarrow\left(\Sigma_{j} \backslash O\right) \times \mathbb{S}^{n-2}$, определенное равенством $\bar{q}(t)=\left(\varphi_{i}(t), q(t)\right)$, удовлетворяет условию $\varphi_{i}=P \bar{q}=\Phi \bar{q}, t \in \mathbb{S}_{i}^{1}$. Отображение $\bar{q}$ будет кусочно линейным, если $q$ выбрано кусочно линейным. Поскольку $\bar{q}-$ вложение, то мы построили вложение каждой из $s$ дуг на окружности $\mathbb{S}_{i}^{1}$ в границу $\partial W^{n}(V)$ с точностью до нужных смежных стыковок.

Каждое из четырех выражений $\left(\Sigma_{j} \backslash O\right) \times \mathbb{S}^{n-2}$ (здесь $\mathbb{S}^{n-2}=\partial Q^{n-1}, Q^{n-1}=$ $\left.Q^{n-2} \times[-1,1]\right)$ содержит произведение $\left(\Sigma_{j} \backslash O\right) \times \mathbb{S}^{n-3}\left(\right.$ здесь $\left.\mathbb{S}^{n-3}=\partial Q^{n-2}\right)$, которое по непрерьвности продолжается до $\Sigma_{j} \times \mathbb{S}^{n-3}$. Сфера $O \times \mathbb{S}^{n-3}$ является общей для всех четырех выражений $\left(\Sigma_{j} \backslash O\right) \times \mathbb{S}^{n-2}, j=1, \ldots, 4$. Стыковки $s$ дуг $l_{i p}$ должны происходить в точках сферы $O \times \mathbb{S}^{n-3}$. Согласование смежных стыковок для фиксированной окружности $\mathbb{S}^{1} \in\left\{\mathbb{S}_{i}^{1}, i=1,2, \ldots, 16\right\}$ проведем следуюшим образом. Выберем $s$ стыковочных точек на сфере $O \times \mathbb{S}^{n-3}$ для заданной окружности $\mathbb{S}^{1}$ и каждое отображение $q: \operatorname{Int}\left(l_{i p}\right) \rightarrow \mathbb{S}^{n-2}$ зададим так, чтобы образ дуги $q\left(l_{i p}\right)$ соединял нужные стыковочные точки на сфере $O \times \mathbb{S}^{n-3}$. При этом все внутренние точки дуги $q\left(l_{i p}\right)$ должны находиться в $\mathbb{S}^{n-2}$ и только точки стыковки должны находиться на сфере $O \times \mathbb{S}^{n-3}$. Проводя аналогичные рассуждения для всех $s$ дуг и используя последовательно построенные отображения $\widetilde{\varphi}_{i}\left(l_{i p} \times 1\right) \rightarrow\left(\Sigma_{j} \times O\right) \times \mathbb{S}^{n-2}$, мы получаем окончательное вложение $\widetilde{\varphi}_{i}\left(\mathbb{S}^{1} \times 1\right) \rightarrow \partial W^{n}(V)$

В результате мы получаем $16 \mathrm{PL}$-гомеоморфных вложений $\widetilde{\varphi}_{i}\left(\mathbb{S}^{1} \times 1\right) \rightarrow \partial W^{n}(V)$ с нужными свойствами: их образы не пересекаются и для каждого $t \in \mathbb{S}^{1}$ точ- 
ки $x=\widetilde{\varphi}_{i}(t)$ и $y=\varphi_{i}(t)$ соединены в $W^{n}(V)$ отрезком $[x, y]$. Продолжая линейно отображение на эти отрезки, мы получаем 16 требуемых PL-отображений $\widetilde{\varphi}_{i}\left(\mathbb{S}^{1} \times[0,1]\right) \rightarrow W^{n}(V)$. Ретракция $W^{n}(V)$ в $V$ переводит каждое из этих отображений в отображение окружности, реализующее соответствуюшее соотношение представления. При этом $\widetilde{\varphi}_{i}\left(\mathbb{S}^{1} \times 1\right) \rightarrow \partial W^{n}(V)$ и $\widetilde{\varphi}_{i}\left(\mathbb{S}^{1} \times O\right) \rightarrow V$.

Будем называть образы отображений $\widetilde{\varphi}_{i}\left(\mathbb{S}^{1} \times[0,1]\right) \rightarrow W^{n}(V)$ полосками и обозначать их $q_{i}$. Каждую полоску можно представлять как цилиндр отображения $\varphi_{i}$, отвечающего соответствуюшему соотношению $F_{i}, \quad 1 \leqslant i \leqslant 16$. Обозначим объединение этих полосок $\bigcup_{i=1}^{16} q_{i}$ через $T$. Крайнюю окружность полоски $q_{i}, \quad i=1, \ldots, 16$, лежащую на гранище $W^{n}(V)$, т.е. $\widetilde{\varphi}_{i}\left(\mathbb{S}^{1} \times 1\right)$, обозначим $\bar{\Sigma}_{i}^{1}$.

в) Построим ручки, отвечаюшие соотношениям $F_{i}$, чтобы получить многообразие $\bar{W}^{n}(\pi)$.

Сначала построим PL-отображение диска $D_{i}^{2}$ в $\mathbb{R}^{n}, n \geqslant 5$, образ которого служит продолжением полоски $q_{i}$. Для этого PL-отождествим граничную окружность диска $D_{i}^{2}$ с внешней PL-окружностью $\bar{\Sigma}_{i}^{1}$ и продолжим это отображение до вложения диска $D_{i}^{2}$ в пространство $\mathbb{R}^{n}$.

В силу того, что $W^{n}(V)$ является регулярной окрестностью одномерного комплекса $V$, приводя отображение $D_{i}^{2} \rightarrow \mathbb{R}^{n}$ в общее положение, мы получим вложение диска $D_{i}^{2}$ в $\overline{\mathbb{R}^{n} \backslash W^{n}}$, продолжающее полоску $q_{i}$. Будем обозначать этот образ по-прежнему $D_{i}^{2}$. Он не пересекает другие аналогичные диски и пересекает крендель $W^{n}(V)$ только по ломаной окружности $\bar{\Sigma}_{i}^{1}$.

Объединение $V \cup T \cup \bigcup_{i=1}^{16} D_{i}^{2}$ есть, очевидно, полиэдр $P^{2}(\pi)$ представления $\pi$.

Построим теперь в $\mathbb{R}^{n} 16$ ручек, приклеенных к $W^{n}(V)$, остовными дисками для которых являются построенные 2 -диски $D_{i}^{2}$. Заметим, что приклеивание каждого диска $D_{i}^{2}$ к крендельному многообразию $W^{n}(V)$ является локально трансверсальным в кусочно линейной категории. Это означает, что каждая точка $x \in \bar{\Sigma}_{i}^{1}$ имеет окрестность $U_{x}^{n-1}$ на поверхности кренделя $W^{n}(V)$ и окрестность $V_{x}^{2}$ на диске $D_{i}^{2}$ такие, что $U_{x}^{n-1} \cap V_{x}^{2}=\bar{\Sigma}_{i}^{1} \cap V_{x}^{2}$ и пара $\left(U_{x}^{n-1}, V_{x}^{2}\right)$ PL-гомеоморфна стандартной паре дисков $\left(Q^{n-1}, Q_{+}^{2}\right)$ в полупространстве $\mathbb{R}_{+}^{n}$ (см. рис. 3 ).

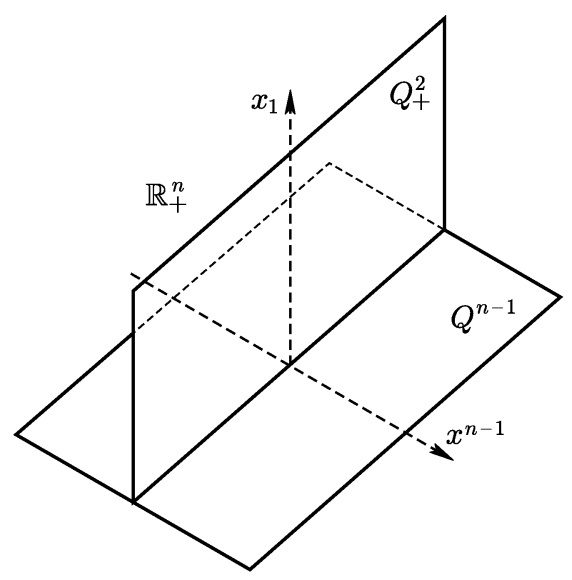

Рис. 3. Пара клеток $\left(Q^{n-1}, Q_{+}^{2}\right)$ 
Теперь можно взять специальную регулярную окрестность каждого диска $D_{i}^{2}$ в $\mathbb{R}^{n}$ nо модулю крендельного многообразия $W^{n}(V)$. Это означает, что мы берем симплициальную окрестность диска $D_{i}^{2}$ во втором барицентрическом подразделении триангуляции пространства $\mathbb{R}^{n}$, для которой $\partial W^{n}$ и $D^{2}$ являются подкомплексами. При этом мы не используем симплексы, пересекающиеся с внутренностью многообразия $W^{n}$. Эту регулярную окрестность обозначим $N^{n}\left(D_{i}^{2}\right)$. Согласно теореме W3 симплициальная окрестность $N^{n}\left(D_{i}^{2}\right)-n$-мерная клетка, так как $N^{n}\left(D_{i}^{2}\right) \searrow D_{i}^{2} \searrow o$, где $o$ - некоторая точка диска $D_{i}^{2}$. Пересечение $\partial W^{n} \cap N^{n}\left(D_{i}^{2}\right)$ - регулярная окрестность подошвенной окружности $\bar{\Sigma}_{i}^{1}=\partial D_{i}^{2}$ в крае $\partial W^{n}(V)$. Обозначим ее $N^{n-1}\left(\bar{\Sigma}_{i}^{1}\right)$. Имеем $N^{n}\left(D_{i}^{2}\right) \searrow D_{i}^{2}$, а также то, что окрестность $N^{n-1}\left(\bar{\Sigma}_{i}^{1}\right)$ PL-окружности $\bar{\Sigma}_{i}^{1}$ комбинаторно стягивается на эту окружность.

Пара $\left(N^{n}\left(D_{i}^{2}\right), D_{i}^{2}\right), n \geqslant 5$, PL-эквивалентна стандартной паре клеток $\left(Q^{n}, Q^{2}\right), \quad n \geqslant 5$, что доказывается PL-теорией [16]. Иначе говоря, пара $\left(N^{n}\left(D_{i}^{2}\right), D_{i}^{2}\right)$ PL-эквивалентна паре $\left(Q^{2} \times Q^{n-2}, D^{2} \times O\right)$. При этом (в силу теоремы W2 о единственности регулярной окрестности) окрестность $N^{n-1}\left(\bar{\Sigma}_{i}^{1}\right)$ изотопно переводится в крае $\partial\left(N^{n}\left(D_{i}^{2}\right)\right)$ в окрестность вида $\partial D_{i}^{2} \times Q^{n-2}$. Следовательно, регулярная окрестность $N^{n}\left(D_{i}^{2}\right)$ - это ручка индекса два $D^{2} \times_{\mathrm{PL}} D^{n-2}$, приклеенная в $\mathbb{R}^{n}$ к многообразию $W^{n}(V)$ по трубчатой окрестности $N^{n-1}\left(\bar{\Sigma}_{i}^{1}\right)$ с индуцированной тривиализацией, что и требовалось доказать.

Приклеивая указанным образом все ручки $N^{n}\left(D_{i}^{2}\right)$ по соответствующим подошвенным многообразиям $N^{n-1}\left(\bar{\Sigma}_{i}^{1}\right)$, мы сопоставим выбранному представлению $\pi \in \Pi(4,16)$ следуюшее многообразие:

$$
\bar{W}^{n}(\pi)=\left\{W^{n}(V)\right\} \cup\left\{\bigcup_{i=1}^{16} N^{n}\left(D_{i}^{2}\right)\right\}, \quad \partial D_{i}^{2}=\bar{\Sigma}_{i}^{1}, \quad i=1,2, \ldots, 16 .
$$

г) Докажем, что $\bar{W}^{n}(\pi) \searrow P^{2}(\pi)$.

Сначала покажем, что

$$
\bar{W}^{n}(\pi) \searrow\left(W^{n}(V) \cup \bigcup_{i=1}^{16} D_{i}^{2}\right) \searrow\left(V \cup T \cup \bigcup_{i=1}^{16} D_{i}^{2}\right)=P^{2}(\pi)
$$

т. е. что многообразие $\bar{W}^{n}(\pi)$ - это регулярная окрестность полиэдра данного группового представления $P^{2}(\pi)$.

Согласно теореме $\mathrm{W} 1$ имеем, что $\bar{W}^{n}(\pi) \searrow W^{n}(V) \cup \bigcup_{i=1}^{16} D_{i}^{2}$. Это означает, что каждая ручка $N^{n}\left(D_{i}^{2}\right)$ симплициально стягивается на свой остовной диск $D_{i}^{2}$.

Согласно построению, проведенному в п. а), мы получаем, что крендель $W^{n}(V)$ является комбинаторным конусом отображения $\Phi: \partial W \rightarrow V$. Применяя лемму 1 и полагая $K=\bigcup_{i=1}^{16} \bar{\Sigma}_{i}^{1}$, получаем требуемое (клеточное) комбинаторное стягивание $W^{n}(V) \searrow(V \cup T)$, т.е.

$$
\left(W^{n}(V) \cup \bigcup_{i=1}^{16} D_{i}^{2}\right) \searrow\left(V \cup T \cup \bigcup_{i=1}^{16} D_{i}^{2}\right)
$$

или $W^{n}(\pi) \searrow P^{2}(\pi)$, что и требовалось доказать. 
Следовательно, $\bar{W}^{n}(\pi)$ - регулярная окрестность $N^{n}\left(P^{2}(\pi)\right)=N^{n}(\pi)$ полиэдра $P^{2}(\pi)$ данного представления $\pi$.

2) Построим многообразие Маркова $M^{n}(\pi)$, отвечающее представлению $\pi$. Выберем на поверхности кренделя $W^{n}(V)$ некоторую гиперплоскую клетку $B^{n-1}$ и изолированно друг от друга приклеим к ней четыре стандартные ручки $\widehat{H}_{j}^{n}$ индекса два (см. $\S 2)$. При этом предполагаем, что ручки $\widehat{H}_{j}^{n}$ не пересекаются с 16 ручками $N^{n}\left(D_{i}^{2}\right)$. Подошвенные окружности четырех стандартных ручек $\widehat{H}_{j}^{n}$ обозначим через $\bar{\Sigma}_{0, j}^{1}$, а их подошвенные окрестности-через $N^{n-1}\left(\bar{\Sigma}_{0, j}^{1}\right), j=1, \ldots, 4$.

Назовем заполненным многообразием Маркова, отвечаюшим представлению $\pi \in \Pi(r, m)$ (в рассматриваемом случае $r=4, m=16$ ), многообразие

$$
M^{n}(\pi)=\left\{\bar{W}^{n}(\pi)\right\} \cup\left\{\bigcup_{j=1}^{4} \widehat{H}_{j}^{n}\right\}
$$

Многообразие $\bar{W}^{n}(\pi)=N^{n}(\pi)$ и многообразие Маркова $M^{n}(\pi)$ имеют ту же самую фундаментальную группу, которая задана представлением $\pi$, как и края этих многообразий.

3) Построим вспомогательное многообразие $W_{0}$. Приклеим в $\mathbb{R}^{n} \mathrm{k} \bar{W}^{n}(\pi)$ четыре стандартные ручки $H_{j}^{n}$, которые вместе с $W^{n}(V)$ образуют $n$-мерную клетку. Очевидно, можно рассмотреть $H_{j}^{n}=\widetilde{\Delta}_{j} \times \mathbb{Q}^{n-2}$. Обозначим многообразие $\bar{W}^{n}(\pi) \cup \bigcup_{j=1}^{4} H_{j}^{n}$ через $W_{0}$ и назовем его вспомогательным многообразием.

Подошвенные окружности таких ручек - это $\partial \widetilde{\Delta}_{j}=\widetilde{\Sigma}_{j}^{1}$, а подошвенные окрестности - это $\widetilde{\Sigma}_{j}^{1} \times Q^{n-2}$. Потребуем, чтобы окрестности $\widetilde{\Sigma}_{j}^{1} \times Q^{n-2}$ не пересекались с подошвенными окрестностями $N^{n-1}\left(\bar{\Sigma}_{i}^{1}\right)$ и подошвенными окрестностями четырех ручек $H_{j}^{n}$. (Все указанные окрестности не пересекаются между собой.) Потребуем также, чтобы ручки $H_{j}^{n}$ не пересекались с ручками $N^{n}\left(D_{i}^{2}\right)$, что возможно в силу общего положения.

Таким образом, многообразие $W_{0}$ получается приклеиванием к PL-клетке в $\mathbb{R}^{n}$ шестнадшати ручек индекса 2. Его фундаментальная группа тривиальна.

4) Построим PL-изоморфизм, отображающий $M^{n}(\pi)$ на $W_{0}$, для случая тривиальной фундаментальной группы $\pi$. Пусть представление $\pi$ задает тривиальную группу. Тогда край многообразия $\bar{W}^{n}(\pi)$ односвязен. На этом крае лежат две группы по четыре PL-окружности: $\partial \widetilde{\Delta}_{j}-$ первая группа окружностей, обходящих стандартные “дырки” кренделя $W^{n}(V)$, и $\bar{\Sigma}_{0, j}^{1}$ - вторая группа окружностей, являющихся подошвенными окружностями четырех дополнительных стандартных ручек $\widehat{H}_{j}^{n}$.

Как следует из доказанной леммы 2, эти четверки соответственно изотопны в четырехмерном односвязном многообразии $\partial \bar{W}^{n}(\pi)$. Это многообразие, являясь краем регулярной окрестности, вложено в $\mathbb{R}^{n}$ с двусторонним воротником, т. е. имеет окрестность, PL-изоморфную $\partial \bar{W}^{n}(\pi) \times[-1,1]$, в которой само многообразие $\partial \bar{W}^{n}(\pi)$ отвечает координате 0 . Такая изотопия обычным образом продолжается послойно до изотопии $\mathbb{R}^{n}$, тождественной вне двустороннего воротника.

Эта изотопия переводит многообразие $M^{n}(\pi)$ в многообразие $M^{\prime} \subset \mathbb{R}^{n}$, которое получается из $\bar{W}^{n}(\pi)$ приклеиванием четырех ручек $\left(\widehat{H}_{j}^{n}\right)^{\prime}$, являюшихся образами ручек $\widehat{H}_{j}^{n}$ при построении PL-изотопии. При этом подошвенные окрест- 
ности этих ручек совпадают с подошвенными окрестностями соответствуюших ручек $H_{j}^{n}$.

Многообразие $M^{\prime}$ получается приклеиванием двадщати ручек индекса два к кренделю $W^{n}(V)$. Порядок приклеивания их безразличен, и можно считать, что это многообразие получается приклеиванием шестнадцати ручек к многообразию $W^{\prime}$, которое возникает так же, как при приклеивании четырех ручек к $W^{n}(V)$ по подошвенным окрестностям $\widetilde{\Sigma}_{j}^{1} \times Q^{n-2}$. Очевидно, последнее многообразие является регулярной окрестностью букета четырех двумерных дисков с той же самой точкой склейки, что и у букета $V$, т. е. комбинаторно стягиваемого комплекса, и, следовательно, оно само является PL-клеткой.

Многообразие $M^{\prime}$ получено приклеиванием шестнадщати ручек индекса два к $n$-мерной PL-клетке и PL-гомеоморфно многообразию $W_{0}$. Действительно, pacсмотрим приклеивание одной ручки к $n$-клетке в $\mathbb{R}^{n}$. Многообразие, полученное в результате такого приклеивания, - это регулярная окрестность двумерной сферы, являющаяся объединением двух клеток: остовной клетки ручки и, если $n$-клетка выпукла, клетки, являющейся конусом над подошвенной окружностью ручки из центра $n$-клетки. Согласно результатам, полученным Зиманом, двумерная сфера кусочно линейно не заузливает в пространстве размерности более четыpex [16]. Поэтому в силу единственности регулярной окрестности (теорема W2) результат приклеивания однозначно определен в PL-категории. Значит, с помощю такого приклеивания получаем $S^{2} \times_{\mathrm{PL}} D^{n-2}$. Шестнадщать PL-окружностей в $(n-1)$-мерной сфере при $n \geqslant 5$ лежат в шестнадшати не пересекаюшихся попарно $(n-1)$-мерных клетках. Значит, шестнадцать таких приклеиваний образуют связную сумму шестнадшати прямых произведений $S^{2} \times_{\mathrm{PL}} D^{n-2}$. Однако заполненное многообразие Маркова $M^{n}(\pi)$ при тривиальной фундаментальной группе PL-эквивалентно многообразию $M^{\prime}=W_{0}$. Край этого многообразия PL-изоморфен связной сумме шестнадцати прямых произведений $\mathbb{S}^{2} \times \mathbb{S}^{n-3}$, $16 \#\left(\mathbb{S}^{2} \times \mathbb{S}^{n-3}\right), n \geqslant 5$. Теорема доказана.

ТЕОрема 2. Предположсим, что $\pi$ - задание из класса $\left\{G_{m}\right\}$, которое отвечает нетривиальной группе. Тогда соответствующее многообразие Маркова $M^{n}(\pi)$ имеет край, $\mathrm{PL-негомеоморфный} \mathrm{многообразию} 16 \#\left(\mathbb{S}^{2} \times \mathbb{S}^{n-3}\right)$, $n \geqslant 5$

ДокАЗАТЕЛЬСТВо очевидно, поскольку фундаментальная группа прямого произведения сфер размерности больше 1 тривиальна.

ТЕОРЕМА 3. PL-многообразие $16 \#\left(\mathbb{S}^{2} \times \mathbb{S}^{n-3}\right), \quad n \geqslant 5$, алгоритмически не распознается.

ДокаЗАТЕльство. Докажем, что если PL-многообразие $16 \#\left(\mathbb{S}^{2} \times \mathbb{S}^{n-3}\right)$, $n \geqslant 5$, алгоритмически распознается, то в классе $\left\{G_{w}\right\}$, т.е. во множестве $\Pi(4,16)$-представлений, алгоритмически распознается задание тривиальной группы. Рассмотрим представление $\pi \in G_{w}$ и построим (алгоритмически) по нему заполненное PL-многообразие Маркова $M^{n}(\pi), \quad n \geqslant 5$. Заметим, что в силу теоремы 1 многообразие Маркова $M^{n}(\pi)$ PL-гомеоморфно многообразию $N^{n}(\pi) \cup \bigcup_{j=1}^{4} H_{j}^{n}$. Последнее многообразие легко алгоритмически строится в пространстве $\mathbb{R}^{n}, \quad n \geqslant 5$. По теоремам 1 и 2 многообразие $\partial M^{n}(\pi)$, $n \geqslant 5$, тогда и только тогда имеет тривиальную фундаментальную группу, когда многообразие $\partial M^{n}(\pi), n \geqslant 5$, PL-гомеоморфно многообразию $16 \#\left(\mathbb{S}^{2} \times \mathbb{S}^{n-3}\right)$, 
$n \geqslant 5$. Если предположить, что многообразие $16 \#\left(\mathbb{S}^{2} \times \mathbb{S}^{n-3}\right), \quad n \geqslant 5$, алгоритмически распознается, то в классе $\left\{G_{w}\right\}$ алгоритмически распознается задание тривиальной группы, что противоречит теореме Адяна-Рабина. Теорема доказана.

ЗАМЕЧАнИЕ. С помощю группы Борисова [8] нетрудно построить группу с неразрешимой проблемой тождества такую, что она имеет любое число $N \geqslant 12$ соотношений и некоторое число $N_{0} \leqslant N$ образующих. Тогда из приведенного вьше доказательства теоремы 3 следует, что для любого $N+4 \geqslant 16$ PL-многообразие $(N+4) \#\left(\mathbb{S}^{2} \times \mathbb{S}^{n-3}\right), n \geqslant 5$, алгоритмически не распознается.

Для $1 \leqslant k \leqslant 15$ вопрос о том, будет ли $\mathrm{PL}$-многообразие $k \#\left(\mathbb{S}^{2} \times \mathbb{S}^{n-3}\right), n \geqslant 5$, алгоритмически не распознаваемо, остается открытым.

В $\oint 7$ показывается, что многообразие $14 \#\left(\mathbb{S}^{2} \times \mathbb{S}^{2}\right)$ алгоритмически не распознается. Таким образом, этот вопрос открыт для $k$ таких, что $1 \leqslant k \leqslant 13$.

\section{§6. Алгоритмическая нераспознаваемость гомотопического типа}

Аналогично проблеме распознаваемости комбинаторного изоморфизма можно поставить ряд проблем алгоритмической распознаваемости. Среди них отметим проблемы алгоритмической распознаваемости диффеоморфного, гомеоморфного и гомотопического типов.

Проблему нераспознаваемости диффеоморфного типа мы не можем здесь изложить в силу того, что это потребует существенного увеличения объема настоящей статьи. Алгоритмическая нераспознаваемость диффеоморфного типа доказана в работе [5], где авторы применили разработанную технику эффективного задания гладких многообразий с помощью локальных карт.

Следуюшая теорема легко вытекает из доказанных результатов.

Tеорема 4. Топологический тип PL-многообразия $16 \#\left(\mathbb{S}^{2} \times \mathbb{S}^{n-3}\right), n \geqslant 5$, алгоритмически не распознается.

ДокаЗАтельство. Сначала заметим, что в теореме 1 термин "PL-гомеоморфно" можно заменить на термин "гомеоморфно" и теорема 1 останется справедливой. Далее, в теореме 2 выражение "PL-негомеоморфно" можно заменить на "негомеоморфно". Итак, справедливо следуюшее утверждение. В классе $n$-мерных $\mathrm{PL}$-многообразий топологический тип PL-многообразия $16 \#\left(\mathbb{S}^{2} \times \mathbb{S}^{n-3}\right), n \geqslant 5$, алгоритмически не распознается. Теорема доказана.

Напомним, что все двумерные многообразия алгоритмически классифицируются. Однако вопрос об алгоритмической классификации трехмерных многообразий до сих пор не разрешен. Используя теорему об $h$-кобордизме $(n \geqslant 5$ - теорема $\Phi$ ридмана; $n \geqslant 6$ - теорема Смейла [15]), можно усилить некоторые полученные здесь результаты.

А. Верна теорема об алгоритмической нераспознаваемости топологического типа PL-многообразия $12 \#\left(\mathbb{S}^{2} \times \mathbb{S}^{n-3}\right), n \geqslant 5$.

Б. Верна теорема об алгоритмической нераспознаваемости PL-гомеоморфного типа PL-многообразия $12 \#\left(\mathbb{S}^{2} \times \mathbb{S}^{n-3}\right), n \geqslant 6$.

Вопрос об алгоритмической нераспознаваемости PL-гомеоморфного типа PL-многообразия $12 \#\left(\mathbb{S}^{2} \times \mathbb{S}^{2}\right)$ остается открытым.

Теперь перейдем к вопросам алгоритмической классификации гомотопических типов конечных компактных симплищиальных полиэдров. 
ЛЕмма 3. Пусть $P^{2}$ - конечный двумерный полиәдр с тривиальной фундаментальной группой. Тогда полиәдр $P^{2}$ гомотопически эквивалентен букету $k \vee \mathbb{S}^{2} k$ двумерных сфер, где $k$ - число образующих свободной абелевой әруппы $H_{2}\left(P^{2}, \mathbb{Z}\right)$.

ДоКАЗАТЕЛЬСТВО. Известно, что $n$-мерная группа целочисленных гомологий $n$-мерного полиэдра является свободной абелевой группой. Пусть $k$ - число образующих групшы $H_{2}\left(P^{2}, \mathbb{Z}\right)$. Одномерная группа гомологий любого полиэдра изоморфна его прокоммутированной фундаментальной группе; следовательно, $H_{1}\left(P^{2}, \mathbb{Z}\right)=0$. По теореме Гуревича [17]-[19] вторая гомотопическая группа $\pi_{2}\left(P^{2}, x_{0}\right)$ полиэдра $P^{2}$ изоморфна группе $H_{2}\left(P^{2}, \mathbb{Z}\right)$. Таким образом, существует отображение $f:\left(k \vee \mathbb{S}^{2}\right) \rightarrow P^{2}$, являюшееся геометрической реализацией системы образуюших второй гомотопической группы $\pi_{2}\left(P^{2}, x_{0}\right)$ с базисной точкой $x_{0}$, при этом базисная точка букета переходит в базисную точку $x_{0}$ полиэдра $P^{2}$. Имеются изоморфизм $\pi_{2}\left(P^{2}, x_{0}\right) \simeq H_{2}\left(P^{2}, \mathbb{Z}\right)$ и, следовательно, изоморфизм $H_{2}\left(k \vee \mathbb{S}^{2}, \mathbb{Z}\right) \simeq H_{2}\left(P^{2}, \mathbb{Z}\right)$. Высшие группы гомологий полиэдров $k \vee \mathbb{S}^{2}$ и $P^{2}$ тривиальны.

Таким образом, отображение $f$ индуцирует изоморфизм всех групп гомологий полиэдра $k \vee \mathbb{S}^{2}$ в полиэдр $P^{2}$, а так как они оба односвязны, то по теореме Уайтхеда [17]-[19] отображение $f$ является гомотопической эквивалентностью. Лемма доказана.

ТЕОРема 5. Гомотопический тип (простой гомотопический тип) букета двумерных сфер $12 \vee \mathbb{S}^{2}$ алгоритмически не распознается в классе двумерных полиэдров.

ДоКАЗАТЕЛЬСТВО. Предположим, что гомотопический тип двумерного полиэдра $12 \vee \mathbb{S}^{2}$ алгоритмически распознается. Рассмотрим класс $\left\{G_{w}\right\}$, состояший из П $(4,16)$-групп (см. $\S 1)$, и какую-либо П $(4,16)$-группу из $\left\{G_{w}\right\}$ и построим для нее полиэдр $P^{2}(4,16)$. Число $k$ легко вычисляется для полиэдра $P^{2}(4,16)$, оно равно 12 . По лемме 3 полиэдр $P^{2}(4,16)$ тогда и только тогда гомотопически эквивалентен букету $12 \vee \mathbb{S}^{2}$, когда полиэдр $P^{2}(4,16)$ имеет тривиальную фундаментальную группу. Если гомотопический тип букета $12 \vee \mathbb{S}^{2}$ алгоритмически распознается, то алгоритмически распознается, будет или не будет всякий полиэдр $P^{2}(4,16)$ иметь тривиальную фундаментальную группу. Это противоречит теореме Адяна-Рабина. Таким образом, не существует алгоритма для распознавания гомотопического типа двумерного полиэдра $12 \vee \mathbb{S}^{2}$. Теорема доказана.

Tеорема 6. Гомотопический тип (простой гомотопический тип) полиэдра $\left(12 \vee \mathbb{S}^{2}\right) \times I^{n-2}$ алгоритмически не распознается в классе полиэдров размерности не более $n$.

ДоКАЗАТЕЛЬСТвО. Действительно, если предположить, что гомотопический тип полиэдра $\left(12 \vee \mathbb{S}^{2}\right) \times I^{n-2}$ алгоритмически распознается, то, поскольку при умножении пространства $X$ на куб произвольной размерности гомотопический тип пространств $X$ и $X \times I^{k}$ одинаков, распознается гомотопический тип полиэдра $12 \vee \mathbb{S}^{2}$, что противоречит теореме 5 . Теорема доказана.

ТЕорема 7. Общая проблема нахождения алгоритма для ответа на вопрос, будут ли два произвольных конечных полиэдра иметь одинаковье или разные гомотопические типь (простые гомотопические типь), алгоритмически неразрешима. 
ДокАЗАТЕЛЬСТво. Действительно, можно считать, что теорема 6 доказана для любого $n \geqslant 2$. Следовательно, гомотопический тип полиэдра $12 \vee \mathbb{S}^{2}$ алгоритмически не распознается в классе всех конечных полиэдров. Теорема доказана.

\section{§7. Дополнения, комментарии, замечания}

1. Вместо сферической перестройки (замены в шаре $B^{4}$ с 1 -ручками туннеля $\mathbb{S}^{1} \times D^{3}$ на многообразие $\left.D^{2} \times \mathbb{S}^{2}\right)$ А. А. Марков применял свою конструкцию: замену туннелей на их дополнения в сфере $\mathbb{S}^{4}$. Поскольку окружность не заузливает в $\mathbb{S}^{4}$, эти две конструкции, по существу, эквивалентны, причем конструкция А. А. Маркова имеет то преимущество, что не требуется выбор тривиализации нормальных трубчатых окрестностей окружностей.

2. Возможность алгоритмического построения многообразия Маркова (используемого в настоящей статье) по имеющемуся заданию группы не вызывает сомнения. Однако, поскольку в теореме 3 речь идет об алгоритмической нераспознаваемости многообразия, нет смысла указывать степень сложности этого алгоритма или его компьютерную реализацию.

3. Используя результаты работы [23] и группу Борисова [8], нетрудно снизить число слагаемых $\mathbb{S}^{2} \times \mathbb{S}^{2}$ в нераспознаваемой связной сумме $k \# \mathbb{S}^{2} \times \mathbb{S}^{2}$ до четырнадшати. Таким образом, в настоящее время минимальное нераспознаваемое многообразие - это $14 \#\left(\mathbb{S}^{2} \times \mathbb{S}^{2}\right)$.

4. Краткое изложение теоремы Маркова имеется в работе [24].

5 . В работе [25] приводится некоторое уточнение числа $k$ слагаемых в нераспознаваемом многообразии $k \#\left(\mathbb{S}^{2} \times \mathbb{S}^{2}\right)$, сделанное А. Б. Сосинским.

6. Отметим интересную недавно появившуюся работу [26].

\section{Список литературы}

1. Марков A. A. Insolubility of the problem of homeomorphy // Proc. Intern. Congress of Math. Cambrige: Cambrige Univ. Press, 1960. P. 300-306.

2. Марков А. А. Неразрешимость проблемы гомеоморфии // ДАН СССР. 1958. Т. 121. № 2. C. 218-220.

3. Марков А.А. Неразрешимость проблемы гомеоморфии // УМН. 1958. Т. 13. №4. C. $213-216$.

4. Зейферт Г., Трельфалль В. Топология. М.: ГОНТИ, 1938.

5. Boone $W$. W., Haken $W$., Poenaru $V$. On recursively usolvable problems in topology and their classification // Contrib. to Math. Logic. Amsterdam: North-Holland. Publ. Comp., 1968. P. 37-74.

6. Milnor J. A procedure for killing homotopy groups of differentiable manifolds // Proc. Symp. Pure Math. 1961. № 3. P. 39-55.

7. Новиков С. П. Гомотопически эквивалентные гладкие многообразия. I // Изв. АН СССР. Сер. матем. 1964. Т. 28. № 2. С. 365-474.

8. Борисов В. В. Простые примеры групп с неразрешимой проблемой тождества // Матем. заметки. 1969. Т. 6. С. 521-532.

9. Адян С.И. Алгоритмическая неразрешимость проблем распознавания некоторых свойств групп // ДАН СССР. 1955. Т. 103. № 4. С. 533-535.

10. Адян С. И. Неразрешимость некоторых алгоритмических проблем теории групп // Тр. MMO. 1957. T. 6. C. 231-298.

11. Адян С. И., Дурнев И. Г. Алгоритмические проблемы для групп и полугрупп // УМН. 2000. T. 55. № 2. С. 3-94.

12. Rabin M.O. Recursive unsolvability of group theoretic problems // Ann. Math. 1958. V. 67. P. $172-194$. 
13. Freedman M. H. The Topology of four-dimensional manifolds // J. Diff. Geom. 1982. V. 17. P. 357-453.

14. Siebenmann L. C. La Conjecture de Poincare Topologique en dimension 4 // Seminare Bourbaki. 1981/82. № 588 .

15. Freedman M. H., Quinn F. S. Topology of 4-manifolds // Princeton Math. Series. V. 39. Princeton-N.J.: Princ. Univ. Press, 1990.

16. Рурж К. П., Сандерсон Б. Дж. Введение в кусочно линейную топологию. М.: Мир, 1974.

17. Ху С.-ЦL. Теория гомотопий. М.: Мир, 1974.

18. Хилтон П., Уайли С. Теория гомологий. Введение в алгебраическую топологию. М.: Мир, 1966.

19. Гриффитс Ф.А., Морган Д. Б. Рациональная теория гомотопий и дифференциальные формы. М.: Наука, 1990.

20. Whitehead J. H. C. Simplicial spaces nuclei and $m$-groups // Proc. Lond. Math. 1939. V. 45. P. 243-327.

21. Lickorich W.B.R., Siebenmann L.C. Regular neighbourhoods and the stable range // Trans. AMS. 1969. V. 139. P. 207-230.

22. Higman G., Neumann B. H., Neumann H. Embedding theorem for groups // J. Lond. Math. Soc. 1949. V. 24. P. 247-254.

23. Miller C. F. III. Algorithms and Classification in Combinatorial Group Theory // Math. Sci. Res. Pub. V. 23. N.Y.: Springer, 1992. P. 1-59.

24. Фоменко A.T. Дифференциальная геометрия и топология. Дополнительные главы. Ижевск: Ижевская республиканская типография, 1999.

25. Володин H. А., Фоменко А. Т. Многообразия, узлы, алгоритмы // Тр. семинара по векторному и тензорному анализу. 1978. № 18. С. 94-128.

26. Лексин В. П, Чернавский A. В. Нераспознаваемость многообразий. К теореме С. П. Новикова о нераспознаваемости сферы $\mathbb{S}^{n}$ при $n \geqslant 5 / /$ Докл. РАН 2003. Т. 391. № 4 . C. $453-455$. 